\title{
Examining the Linguistic Equivalency and Cross-Cultural Adaptation of the Sensory Processing and Self-Regulation Checklist- Tagalog Version
}

\author{
Ivan Neil Benitez Gomez ${ }^{1,2}$, Pauline Grace G. Morato-Espino 1,2, \\ Cynthia YY Lai ${ }^{3}$ \\ ${ }^{1}$ Center for Health Research and Movement Science, University of Santo Sto. Tomas, Manila, Philippines \\ ${ }^{2}$ Department of Occupational Therapy, College of Rehabilitation Sciences, University of Santo Sto. Tomas, Manila, Philippines \\ ${ }^{3}$ Department of Rehabilitation Sciences, The Hong Kong Polytechnic University, Kowloon, Hong Kong SAR
}

\begin{abstract}
Children's ability to adequately process sensory information from their environments contribute to the development of self-regulation. The increasing prevalence of sensory processing difficulties in both clinical and normative childhood population underscores the need for assessment. However, in the Philippine context, there is no crossculturally validated tool that measures sensory processing and self-regulation among children. Both language equivalency and cultural-relevance should be addressed when translating health-related outcomes. In this study, the accuracy of linguistic equivalency and cultural relevance of the Tagalog version of the Sensory Processing and Self-Regulation Checklist (SPSRC) was validated. Using a multi-step process of forward translation, equivalence of translation testing, backward translation, face, and content validation, and cross-cultural adaptation inquiry, the SPSRC-Tagalog was examined. Adaptation of colloquial English terms was incorporated, and several items whose examples were deemed not culturally relevant were modified to reflect the language and culture it is intended for. The findings in this study support the linguistic equivalency and cultural-relevance of the SPSRC-Tagalog as a single measure of sensory processing and self-regulation abilities of Tagalog-speaking Filipino children that can be used by Filipino occupational therapists. Future studies should further examine its psychometric properties in the target population.
\end{abstract}

Keywords: linguistic equivalence, cross-cultural adaptation, Tagalog version of the Sensory Processing and Self-Regulation Checklist, translation

(Asian J Occup Ther 17: 57-63, 2021)

\section{Introduction}

Sensory processing refers to the ability to detect and organize sensory information from the environment and integrate them in order for appropriate responses to be generated $[1,2]$. The prevalence of sensory processing difficulties among children has been seen in both clinical and general populations. For example, children with neurodevelopmental disabilities, sensory processing difficulties were seen in as much as $80 \%$ [3]; while

Received: 2 July 2020, Accepted: 15 April 2021

Corresponding to: Ivan Neil Benitez Gomez, Rm. 124 St. Martin de Porres Building, University of Santo Tomas, España Boulevard, Sampaloc, Manila, 1015 Philippines

e-mail: ibgomez@ust.edu.ph

(C2021 Japanese Association of Occupational Therapists records have been documented to be between 5-28\% among typically developing children $[4,5]$. Sensory processing difficulties affect children's ability to participate in occupations $[1,2]$, and the development of self-regulator abilities necessary for higher order functional skills [6]. This underscores the importance measuring sensory processing and self-regulation among children. However, in the Philippine context, there is no known cross-culturally relevant and validated tool that may do so.

The adaptation of health-related measurement tools developed in Western countries has been a common worldwide clinical practice [7]. A similar trend can also be observed among developing countries, such as the Philippines $[8,9]$. The pressure to adopt an evidencebased practice, the use of psychometrically tested outcome measures, lack of financial and temporal resourc- 
es, and growing interest in cross-cultural comparisons among normative and clinical groups have motivated this paradigm shift $[7,10]$.

The most basic process of cross-cultural adaptation of health-related measurement tools involves translation from the original text to the intended language of use [11-13]. However, this mere translation may pose to be more problematic than helpful. The existence of culturedependent concepts may lead to misinterpretation when items on a tool are translated into the target language $[8,10]$. Literal translations may disregard language form and content. Thus, when a translation is hastily performed, the original meaning behind the items may change, alongside its intended constructs and its psychometric properties [7, 10-13].

Translation of health-related measurement tools entails the careful translation of their meaning into the target language. Language equivalency is a suggested criterion that should be mindfully integrated into the translation procedures. Several translation procedures can be seen in the literature, but the most common methods involve forward and backward translation. In forward translation, a bilingual expert translates the original instrument from the original language to the target language. In the backward translation procedure, an independent bilingual expert translates the instrument back to its original form. Experts are then recruited to compare the backward translated instrument with the original one to detect inconsistencies [14-16]. More recently, the use of an expert panel to determine language equivalency has been suggested [16]. This may take several forms. One approach recruits an expert panel that evaluates the forward translated instrument and looks at whether the translated items contain the same meaning within the target language $[11,17]$.

While these approaches are useful, they might be limited to the form and content of the language, and not on the cultural relevance of the items. The physical objects or materials, and the level of familiarity, meaning, and value an individual places on them are not explicitly addressed $[18,19]$. Language and culture, while different constructs, should be considered jointly when adapting health-related measurement tools [11, 13]. Determining the cultural appropriateness and relevance of the items on the translated instrument should be an implicit procedure towards the adaptation of an instrument.

Cross-cultural validation may be performed during the process of translation. For example, Beaton and collegues [11] suggests that translators should be oriented to both language and culture of the target language in order for equivalency of the translation and adaptation. During cross-cultural translations, equivalence of the concept, items, semantics, context, measurement, and function should be considered [12]. This can be achieved by a consensus panel of experts who are knowledgeable of the assessment construct, language, and culture [11]. In determining cross-cultural adequacy, authors may consider the use of quantitative (i.e. survey instruments) and qualitative (i.e., focus group discussions) methods [7, 10-17].

In this study, we examine the linguistic equivalency and cross-cultural adaptation of the Sensory Processing and Self-Regulation Checklist-Tagalog version (SPSRCTagalog) after going a multi-process of translation and language equivalency. In the Philippines, there is no known outcome measure similar to the constructs of the SPSRC-Tagalog. We further employed initial attempts to validate the translated tool using face and content validation tests. Establishing the linguistic equivalency and cross-cultural adaptation of the SPSRC-Tagalog will be useful for Filipino occupational therapists in understanding sensory processing difficulties among Filipino children.

\section{Methods}

Before conducting this research, ethical approval was sought from the University of Santo Tomas- College of Rehabilitation Sciences- Ethics Review Committee. This study reports the preliminary findings on the SPSRC-Tagalog, which is limited to examining its linguistic equivalency and cross-cultural adaptation.

\section{The Sensory Processing and Self-Regulation Checklist (SPSRC)}

The Sensory Processing and Self-Regulation Checklist was developed as a singular tool that can simultaneously measure self-regulation and sensory processing skills among children ages $3-8[18,20]$. It was developed through a rigorous process that involved child development experts that resulted in the current 132-item checklist. The SPSRC has undergone psychometric property testing, and results indicate satisfactory reliability (internal consistency, intrarater reliability) and validity (factor analysis, convergent validity, discriminant validity) $[18,20]$. It is divided into two parts: Self-regulation ability (Part 1) and Sensory processing ability (Part 2). Part 1 is subdivided into three scales (physiological, social/cognitive/emotional, facing changes or challenges), while Part 2 has six sensory-related subscales (auditory, visual, tactile, gustatory/olfactory, vestibular, proprioceptive). Parents are instructed to rate their child's typical performance on the items within the past three months using a 5-point Likert scale (never $=5$, seldom $=4$, sometimes $=3$, most of the time $=2$, always $=1$; reversed scoring for some), where a lower score in- 
dicating performance that is less favorable. The original version is in Chinese [18, 20], but a translated English version is likewise available [21]. The SPSRC-English is a translation of the original version by the authors that underwent a rigorous process of forward translation, backward translation, and review by an expert panel.

\section{Procedures}

This research underwent several phases to report on the cross-cultural and linguistic equivalency of the SPSRC-Tagalog version, guided by the recommendations of the World Health Organization [17] and its original author (Lai, personal communication, February 2018).

\section{Forward Translation}

The Philippines is mostly a bilingual (English and Filipino) nation, with Tagalog being one of the most widely spoken languages. The Tagalog translation of the SPSRC was carried out using the recent English version [21]. Two Filipino occupational therapists (OT) performed the forward translation. Both were licensed OTs with $>5$ years of experience in the field of pediatrics, familiar with sensory processing and selfregulation, and knowledgeable of the English language, but whose mother tongue is Tagalog. The translation was performed independently. A consensus meeting between the forward translators and the research team was conducted to review the initially translated tool. During this meeting, each item on the riginal tool was presented, while the individual forward translators presented their translations. The translations for each item were discussed in order to reach consensus. Diverging translation were deliberated, with the research team's primary investigator mediating the discourse. At the end of the meeting, converging and diverging translations, as well as questionable items endorsed by the translators, were resolved and revised to produce the initial draft of the Tagalog version.

\section{Equivalence of Translation}

The equivalence of translation into the Tagalog version was reviewed out by eight panel members, which included 7 OTs and 1 speech-language pathologist. All expert panel members were licensed (or in the case of the speech-language pathologist, carries the certification to practice) Filipino professionals with $>3$ years of current and continued pediatric practice, familiar with sensory processing and self-regulation, and knowledgeable of the English language, but whose mother tongue is Tagalog. These participants were asked to comment on the equivalence of translation of the SPSRC from English to Tagalog using the Equivalence of Transla- tion Tool by Flaherty [22]. Each item was rated using a 3-point scale (a score of 3 means that the item had precisely the same meaning in both versions, a score of 2 means that the item had almost the same meaning in both versions and a score of 1 means that there are different meanings in each language) and commented on by the experts. Items which scored 1 or 2 by any member of the expert panel were reviewed by the research team considering the specific comments and suggestions and were reworded for the purposes of enehancement of clarity of the Tagalog version.

\section{Backward Translation}

The Tagalog version was then translated back to English by two independent Filipino OTs (different from the OTs who performed the Tagalog translation but sharing similar characteristics) who were naïve of the original version. The translation was conducted independently of each other but was subsequently integrated. Items deemed questionable were revised following the suggestions made by the backward translators and by the study team. In order to avoid biased information language ambiguitites, the research team concorded with author of the original SPSRC in order to address determine whether the translated items retained their original constructs. A consensus was reached between the study team the original author on the Tagalog version of the SPSRC.

Face, Content and Cross-cultural Adaptation Validation

The SPSRC-Tagalog version was evaluated on its face, content, and cross-cultural adaptation validity by fifteen Tagalog-speaking Filipino expert panel members, which consisted of three OTs, three physical therapists, three speech-language pathologists, and three parents. All professionals were licensed therapists with $>3$ years of current and continued pediatric practice. Face, content and cross-cultural adaptation validation can be accomplished using quantitative and qualitative methods [7, 10-17]. In this study, face, content and cross-cultural adaptation validity were measured using the questionnaire by Yaghmaei [23], and comments from the experts. The questionnaire allows for measuring and analyzing the extent of a measurement tool's ability to measure what it intends to measure. General face and content validity were determined by asking yes or no questions. Criteria items with $>80 \%$ were retained, while those with lower ratings were considered for revisions. Each scale on the questionnaire was evaluated on its relevance, clarity, simplicity, and ambiguity, using a 4-point Likert scale (the higher the rating, the better the validity score). Items with a mean score of $>3.00$ were noted and revised based on the comments of the experts, 
Table 1 Summary of Culturally-Adapted Terms in SPSRC-Tagalog

\begin{tabular}{cccll}
\hline Part & Section & Item No & \multicolumn{1}{c}{ SPSRC-English } & \multicolumn{1}{c}{ SPSRC-Tagalog } \\
\hline & A & 6 & Radio broadcast at MTR & Anunsyo sa tren \\
\hline B & 2,6 & Torch light & Flashlight \\
\hline & & 3,9 & Rough mat or grass mat & Carpet o banig \\
& C & $5,12,18$ & Ice or hot-water pad & Yelo o mainit na bimpo \\
& 15 & Hand cream & Lotion \\
\hline & 2 & Markers & Pentel pen \\
& D & 3 & Lemon juice & Calamansi juice \\
\hline E & 12 & Roundabout & Merry-go-round (tsubibo) \\
\hline F & 14 & Play-doh & Clay \\
\hline
\end{tabular}

and subsequently integrated into the final draft of the SPSRC-Tagalog.

\section{Results}

\section{Language Equivalence}

We recorded and tallied the individual responses of the participants on each of the items on the SPSRCTagalog and their rating on its cross-cultural adaptation appropriateness. All items on Part 1 (Self-regulation ability) scored a grade of 3 on the Equivalence of Translation Tool [22] and did not receive any dissenting comments, which suggests its endorsement as crossculturally appropriate for the Tagalog-speaking Filipino population. Across all items, there were no comments on the use of colloquially-adapted terms (using English words in due to lack of existing equivalent word or the word loses its meaning when translated into the Filipino language). The experts also endorsed the adapted Tagalog translation, which used non-direct translation. However, we noted eleven items (nine examples) that scored a 1 on language equivalence (means that there are different meanings in each language) and their respective comments across participants in Part 2 (Sensoryprocessing ability), specifically on the objects used as an example. We found that the process of consesnsus between the translators and the study team, the invaluable comments from the expert panel, and discussions with the tool's original authors are useful practices that allowed us to translate them into the target language and culture [11-13]. It was not that the translation was flawed, but rather the experts commented on its cultural relevance. In this research, we refer to these as nonculturally relevant objects. The participants provided similar suggestions on how these objects used in the items may be more culturally relevant. We modified the nine examples and integrated them into the SPSRCTagalog version. In order to retain the original construct and meaning of these items, discussions between the study team and the original authors proved useful and meaningful [13].

For example, Part 2 Section C Items 3, 9 used "rough mat or grass mat" in its colloquial English form (English form retained in the Tagalog version). The suggestion made by the participants was to omit the adapted colloquial term and replace it with "carpet o banig," a more culturally-relevant and familiar object that can be understood by the target audience. Another comment raised by the participants pertain to the objects depicted in Part Section C Item 18 "ice or hot-water pad." The suggestion was to replace it with "yelo o mainit na bimpo." In this example, we see "ice" directly translated into its Tagalog equivalent, "yelo." However, the "hot-water pad," an item that is somewhat familiar in the Filipino culture (closest equivalence is "hot packs"), is not most accessible or widely-used. Instead, the suggestion of using "mainit na bimpo" was made, which, when translated, refers to hot moist towels, traditionally used by parents on their children. These, among other examples, were suggested by the participants, and a summary of these can be seen in Table 1 .

\section{Face Validity}

All participants deemed the SPSRC-Tagalog as a questionnaire that can be easily read and understood (Table 2). A consensus agreed that it is easy to understand (92.86\%) and administer (92.86\%). However, more than half of the participants $(57.14 \%)$ noted the presence of grammatical errors.

\section{Content Validity}

Generally, all participants rated the SPSRC-Tagalog as a tool that covers major critical areas in sensory processing and self-regulation that are related to each other with appropriate rating scales (Table 3). However, some participants $(14.29 \%)$ questions whether items under 
Table 2 Summary of SPSRC-Tagalog Face Validation

\begin{tabular}{lr}
\hline \multicolumn{1}{c}{ Criteria } & Rating \\
\hline Text can easily be read & $100 \%$ \\
Instructions are easy to understand & $92.68 \%$ \\
Free from grammatical error & $57.14 \%$ \\
Layout is properly arranged & $85.71 \%$ \\
Can easily be administered & $92.68 \%$ \\
Can easily be answered & $100 \%$ \\
Can easily be administered within a reasonable amount & $85.71 \%$ \\
of time & \\
\hline
\end{tabular}

Table 3 Summary of SPSRC-Tagalog General Content Validation

\begin{tabular}{lr}
\hline \multicolumn{1}{c}{ Criteria } & Rating \\
\hline Covers the necessary major areas needed to be measured & $100 \%$ \\
Major areas covered by the tool relate to each other & $100 \%$ \\
$\begin{array}{l}\text { Items under each are adequately represent the area it falls } \\
\text { under in }\end{array}$ & $85.71 \%$ \\
$\begin{array}{l}\text { Items under each major area are related to each other } \\
\text { Rating scale used in the tool appropriate to the items it is } \\
\text { supposed to measure }\end{array}$ & $100 \%$ \\
\hline
\end{tabular}

Table 4 Summary of SPSRC-Tagalog Section Validation

\begin{tabular}{lcccc}
\hline \multicolumn{1}{c}{ Sections } & Relevance & Clarity & Simplicity & Ambiguity \\
\hline Part 1. Self-regulation abilities & & & & \\
1. Physiological functioning & $3.95 \pm 0.14$ & $3.91 \pm 0.10$ & $4.00 \pm 0.00$ & $3.95 \pm 0.10$ \\
2. Social/Cognitive/Emotion Development & $3.98 \pm 0.05$ & $3.86 \pm 0.21$ & $3.94 \pm 0.11$ & $3.92 \pm 0.11$ \\
3. Facing Transition or Challenges & $3.99 \pm 0.03$ & $3.90 \pm 0.12$ & $3.94 \pm 0.16$ & $3.98 \pm 0.06$ \\
\hline Part 2. Sensory processing abilities & & & & \\
1. Auditory & $4.00 \pm 0.00$ & $3.85 \pm 0.20$ & $3.93 \pm 0.03$ & $3.87 \pm 0.27$ \\
2. Visual & $3.98 \pm 0.06$ & $3.93 \pm 0.14$ & $3.99 \pm 0.02$ & $3.99 \pm 0.03$ \\
3. Tactile & $3.99 \pm 0.02$ & $3.91 \pm 0.14$ & $3.99 \pm 0.22$ & $3.99 \pm 0.02$ \\
4. Gustatory/olfactory & $4.00 \pm 0.00$ & $3.92 \pm 0.15$ & $3.92 \pm 0.15$ & $3.95 \pm 0.15$ \\
5. Vestibular & $4.00 \pm 0.00$ & $3.94 \pm 0.08$ & $3.99 \pm 0.02$ & $3.98 \pm 0.04$ \\
6. Proprioceptive & $4.00 \pm 0.00$ & $4.00 \pm 0.00$ & $4.00 \pm 0.00$ & $4.00 \pm 0.00$ \\
\hline
\end{tabular}

each area adequately represent the area it falls under in.

We rated each scale on the SPSRC-Tagalog on its relevance, clarity, simplicity, and ambiguity (Table 4). For the relevance criteria, items on four out of the nine scales (auditory, gustatory/olfactory, vestibular, proprioceptive) were rated as very relevant $(4.00 \pm 0.00)$, with the rest ranging from a mean score of 3.95 to 3.99 . Only the items on the proprioceptive scale were perfectly rated very clear $(4.00 \pm 0.00)$, while the rest ranged from 3.85 to 3.94 . Items on two scales (physiological functioning, proprioceptive processing) were perfectly deemed very simple in its presentation (4.00 \pm 0.00$)$, and the rest ranged from 3.92 to 3.99 . The meaning of the items is most clear (ambiguity) for the proprioceptive scale $(4.00 \pm 0.00)$ compared to the rest, which spanned between 3.87 to 3.99 ).

\section{Discussion}

The SPSRC-Tagalog has undergone a multi-process of translation and linguistic equivalency examination. The Tagalog version of the SPSRC depicted in this study has proven to be an excellent equivalent to the original English version, and is seen to be appropriate for psychometric testing among Tagalog-speaking Filipinos.

In the translation of the SPSRC from English and Tagalog, several English terms were retained and received no objections from the expert panel. The habit of using English integrated into the Tagalog language is a cultural phenomenon observed in the Philippines, a generally considered bilingual nation [24]. This form of communication, seen in both written and oral forms, places standard English words or phrases with the standard Tagalog. A kind of switching occurs between English and Tagalog within and between sentences. Several reasons may exist to explain such practice. Still, in this case, it surmised that the acceptance of the use of English colloquial words or phrases within a Tagalog version of the questionnaire might likely be for precision. Communicative proficiency is preferred over literal translations, which may compromise construct-specific lexical terms from the original source language $[25,26]$. This practice is not exclusive to the SPSRC-Tagalog and can be seen in other Tagalog version of health-related outcome measures, especially among the intended constructs within $[27,28]$. The Tagalog-English codeswitching seen in this study reflects the bilingual nature of the target population the questionnaire is designed for. Further investigation is needed if SPRC is to be translated into other international or local languages.

More than language equivalency, this paper places importance on the cultural-relevance of items in a questionnaire. In the process of examining the equivalency of translation of SPSRC into Tagalog, our experts focused 
on recommending changing the objects of examples in some of the items. For the respondent to provide the best response to a questionnaire item, the construct of the question should be appropriate and relevant to them, in terms of culture and language [29]. Careful consideration of both physical and nonphysical characteristics of the sensory stimulus depicted in the questionnaire is essential to gather the appropriate response $[18,19]$. Future researchers who intend to translate the SPSRC in other languages should look beyond the language translation, and likewise incorporate the relevance of the items within the culture it is designed to be used for.

The preliminary findings on the linguistic equivalance and cross-cultural adapatation of the SPSRCTagalog reported herein are part of a larger study that will examine the psychometric properties of the said tool. Nevertheless, in this research, we demonstrate the quality of the process of translating and determining the language equivalency of the SPSRC-Tagalog. However, there are some limitations in methodology. Whereas we used methods and strategies described in previous literature, the psychometric properties of the SPSRC-Tagalog remain to be answered, an objective beyond the scope of this study. There is a neeed for SPSRC-Tagalog to establish its measurement properties, which includes reliability (i.e., test-retest, internal consistency) and validity (i.e., criterion validity, construct validity) [30]. In the process of translating assessment tools from one language to another, researchers often run the risk of altering the intended meaning of the test items and consequently affecting the validity of the assessment tool. Nevertheless, it is recommended that the SPSRCTagalog be subjected to an extensive sample field testing to evaluate its reliability and validity. This includes an a priori examination of its ecological validity. For this, the bilingual approach suggested [19]. Both the English and Tagalog versions of the SPSRC should be tested on bilingual end users of the questionnaire (i.e. parents and caregivers of children), and their responses compared to determine the translation and language equivalency of the SPSRC-Tagalog in a real-world setting.

\section{Conclusion}

To conclude, these findings in this study support the linguistic equivalency and cultural-relevance of the SPSRC-Tagalog as a single measure of sensory processing and self-regulation abilities of Tagalog-speaking Filipino children. The methods described in this study may serve as a guide in translating the SPSRC in other local languages. Succeeding studies should evaluate its psychometric properties. Using the SPSRC-Tagalog may aid in a better understanding of sensory processing for clinical and research purposes among occupational therapists.

\section{References}

[1] Dunn W. The sensations of everyday life: Empirical, theoretical, and pragmatic considerations. American Journal of Occupational Therapy. 2001; 55(6): 608-20.

[2] Dunn W. Supporting children to participate successfully in everyday life by using sensory processing knowledge. Infants \& Young Children. 2007; 20(2): 84-101.

[3] Cheung PP, Siu AM. A comparison of patterns of sensory processing in children with and without developmental disabilities. Research in Developmental Disabilities. 2009; 30(6): 1468-80.

[4] Ahn RR, Miller LJ, Milberger S, McIntosh DN. Prevalence of parents' perceptions of sensory processing disorders among kindergarten children. American Journal of Occupational Therapy. 2004; 58(3): 287-93.

[5] Gunn TE, Tavegia BD, Houskamp BM, McDonald LB, Bustrum JM, Welsh RK, Mok DS. Relationship between sensory deficits and externalizing behaviors in an urban, Latino preschool population. Journal of Child and Family Studies. 2009; 18(6): 653-61.

[6] Greenspan SI, Wieder S. An integrated developmental approach to interventions for young children with severe difficulties in relating and communicating. Zero to Three. 1997; 17: 5-18.

[7] Bowden A, Fox-Rushby JA. A systematic and critical review of the process of translation and adaptation of generic health-related quality of life measures in Africa, Asia, Eastern Europe, the Middle East, South America. Social Science \& Medicine. 2003; 57(7): 1289-306.

[8] Bernardo AB. Lost in translation? Challenges in using psychological tests in the Philippines. Silliman Journal. 2011; 52: 19-42.

[9] Datu JA. From a rational standpoint: Analyzing nuances in the utility of western psychological tests as assessment tools in the Philippines. International Journal of Research Studies in Psychology. 2012; 2(1).

[10] Schmidt S, Bullinger M. Current issues in cross-cultural quality of life instrument development. Archives of Physical Medicine and Rehabilitation. 2003; 84: S29-34.

[11] Beaton DE, Bombardier C, Guillemin F, Ferraz MB. Guidelines for the process of cross-cultural adaptation of self-report measures. Spine. 2000; 25(24): 3186-91.

[12] Epstein J, Santo RM, Guillemin F. A review of guidelines for cross-cultural adaptation of questionnaires could not bring out a consensus. Journal of Clinical Epidemiology. 2015; 68(4): 435-41.

[13] Van Widenfelt BM, Treffers PD, De Beurs E, Siebelink BM, Koudijs E. Translation and cross-cultural adaptation of assessment instruments used in psychological research with children and families. Clinical child and Family Psychology Review. 2005; 8(2): 135-47.

[14] Hyrkäs K, Appelqvist-Schmidlechner K, Oksa L. Validat- 
ing an instrument for clinical supervision using an expert panel. International Journal of Nursing Studies. 2003; 40(6): 619-25.

[15] Ohrbach R, Bjorner J, Jezewski MA, John MT, Lobbezoo F. Guidelines for establishing cultural equivalency of instruments. New York: University at Buffalo. 2009.

[16] Sousa VD, Rojjanasrirat W. Translation, adaptation and validation of instruments or scales for use in cross-cultural health care research: a clear and user-friendly guideline. Journal of Evaluation in Clinical Practice. 2011; 17(2): 268-74.

[17] World Health Organization. Process of translation and adaptation of instruments. http://www.who.int/substance abuse/research_tools/translation/en/. 2009.

[18] Lai CY, Yung TW, Gomez IN, Siu AM. Psychometric Properties of Sensory Processing and Self-Regulation Checklist (SPSRC). Occupational Therapy International. 2019; 2019.

[19] Neuman A, Greenberg DF, Labovitz DR, Suzuki LA. Cross-cultural adaptation of the Sensory Profile: establishing linguistic equivalency of the Hebrew version. Occupational Therapy International. 2004; 11(2): 112-30.

[20] Lai CY, Chiu AS. Sensory Processing and Self-Regulation Checklist. Heep Hong Society, Hong Kong. 2013.

[21] Lai CY, Chiu AS. Sensory Processing and Self-Regulation Checklist-English Version. Heep Hong Society, Hong Kong. 2019.

[22] Flaherty JA, Gaviria FM, Pathak D, Mitchell T, Wintrob R, Richman JA, Birz S. Developing instruments for cross-cultural psychiatric research. Journal of Nervous and Mental Disease. 1988; 176(5): 257-363.

[23] Yaghmaei, F. Content validity and its estimation. Journal of Medical Education. 2003; 3(1).

[24] Goulet RM. English, Spanish, and Tagalog: a study of grammatical, lexical, and cultural interference. Linguistic Society of the Philippines; 1971.

[25] Bautista ML. Tagalog-English code-switching and the lexicon of Philippine English. Asian Englishes. 1998; 1(1): 51-67.

[26] Bautista ML. Tagalog-English code switching as a mode of discourse. Asia Pacific Education Review. 2004; 5(2): 226-33.

[27] Lipardo DS, Leung AY, Gabuyo CM, Escuadra CJ, Leung PA, Aseron AM, Hernandez KA, Diaz JB, Tsang WW. Cross-cultural adaptation and psychometric properties of the Falls Efficacy Scale-International in Filipino community-dwelling older adults. Disability and Rehabilitation. 2020; 42(9): 1292-8.

[28] Morato-Espino PG, Medallon KG, Benzon R, Arroz MT, Buencamino C, Ibay C, Ko S, Gomez IN. Translation and cross-cultural adaptation of the Friedrich Short Form of the Questionnaire on Resources and Stress (QRS-F) to measure the stress level of Filipino parents and other caregivers of children with disability. Philippine Journal of Allied Health Sciences. 2019; 3(1).

[29] Van de Vijver F, Hambleton RK. Translating tests. European Psychologist. 1996; 1(2): 89-99.

[30] Mokkink LB, Terwee CB, Patrick DL, Alonso J, Stratford PW, Knol DL, Bouter LM, de Vet HC. The COSMIN study reached international consensus on taxonomy, terminology, and definitions of measurement properties for health-related patient-reported outcomes. Journal of Clinical Epidemiology. 2010; 63(7): 737-45. 